Journal of Computer Science 6 (9): 1037-1041, 2010

ISSN 1549-3636

(C) 2010 Science Publications

\title{
Computation of Hahn Moments for Large Size Images
}

\author{
${ }^{1}$ A. Venkataramana and ${ }^{2} \mathrm{P}$. Ananth Raj \\ ${ }^{1}$ Department of Electronics and Communication Engineering, \\ Quli Qutub Shah Government Polytechnic College, Old City, Hyderabad, Andhra Pradesh, India \\ ${ }^{2}$ Department of Electronics and communication Engineering, College of Engineering, \\ Osmania University, Hyderabad-500 007, Andhra Pradesh, India
}

\begin{abstract}
Problem statement: Direct formulas used for Hahn moments computation available in the literature are not applicable for large size images. This is due to the need for calculation of factorial of large values as well as numerical instability in the calculation of polynomial values. This study presents a method to correct these errors. Approach: In this research, recursive formulas for weight function, squared norm and weighted Hahn polynomials that were used for Hahn moments computation were derived. Results: Simulation results were reported for image reconstruction and the quality of reconstructed image was assessed by using universal image quality index. Conclusion: This study presented a procedure for calculation of Hahn moments for any size images using the recursive formulas. From the simulation results, it was observed that the derived recursive formulas work well for any size images.
\end{abstract}

Key words: Weighted Hahn polynomials, Hahn moments, recursive formula

\section{INTRODUCTION}

Orthogonal moments which are extensively used for image reconstruction and classification problems are grouped into either continuous or discrete. Geometric (Hu, 1962), Legendre (Teague, 1980) and Zernike (Teague, 1980) moments are continuous where as Tchebichef (Mukundan et al., 2001), Krawtchouk (Yap et al., 2003) and Hahn moments (Yap et al., 2007) are discrete. Discrete moments have number of advantages over continuous moments like no need for coordinate transformation, better image reconstruction and no discretization error. Recently, Yap et al. (2007) proposed Hahn moments using discrete weighted Hahn polynomials. They showed that Tchebichef and Krawtchouk moments are special cases of Hahn moments with the appropriate parameter settings. In order to compute Hahn moments, the authors used direct formulas for obtaining Hahn polynomials, weight function and squared norm as well as weighted Hahn polynomials that are applicable for small size images only. When these equations are used for large size images, one encounters the following problems (i) Need for calculation of factorial of large values (ii) Numerical instability in the calculation of polynomial values. These problems can be solved by using recursive formulas. Hence, this study derives the recursive formulas for weight function, squared norm and weighted Hahn polynomials. Further, simulation results are reported for image reconstruction using the derived recursive formulas.

\section{MATERIALS AND METHODS}

Direct formulas for weight function, squared norm and Hahn polynomials as given in Table 1 of (Yap et al., 2007) are not suitable for large size images since it requires to calculate the factorial of large values. Further, numerical instability occur in the calculation of polynomial values and therefore, numerical fluctuations occur in the computation of moment values. To overcome these problems, we derive the recursive relation for weight function, squared norm and discrete weighted Hahn polynomials.

\begin{tabular}{lll}
\multicolumn{3}{l}{ Table 1: Computed UIQI for Lena image } \\
\hline & UIQI with Hahn moments & UIQI with Hahn moments \\
Order & for $\left(\alpha_{1}=\alpha_{2}=\beta_{1}=\beta_{2}=0\right)$ & for $\left(\alpha_{1}=\alpha_{2}=\beta_{1}=\beta_{2}=1\right)$ \\
\hline$(20,20)$ & 0.23 & 0.24 \\
$(40,40)$ & 0.43 & 0.44 \\
$(60,60)$ & 0.58 & 0.58 \\
$(80,80)$ & 0.67 & 0.67 \\
$(100,100)$ & 0.75 & 0.76 \\
$(120,120)$ & 0.81 & 0.81 \\
\hline
\end{tabular}

Corresponding Author: A. Venkataramana, Department of Electronics and Communication Engineering, Quli Qutub Shah Government Polytechnic College, Old City, Hyderabad, Andhra Pradesh, India 
Weighted Hahn polynomials (Yap
given by:
$\overline{\mathrm{h}}_{\mathrm{n}}(\mathrm{x} ; \alpha, \beta, \mathrm{N})=\mathrm{h}_{\mathrm{n}}(\mathrm{x} ; \alpha, \beta, \mathrm{N}) \sqrt{\frac{\mathrm{w}(\mathrm{x} ; \alpha, \beta, \mathrm{N})}{\rho(\mathrm{n} ; \alpha, \beta, \mathrm{N})}}$

in which $\mathrm{h}_{\mathrm{n}}(\mathrm{x} ; \alpha, \beta, \mathrm{N})$ are Hahn polynomials which are defined in terms of hypergeometric function as:

$\mathrm{h}_{\mathrm{n}}(\mathrm{x} ; \alpha, \beta, \mathrm{N})={ }_{3} \mathrm{~F}_{2}(-\mathrm{n},(\mathrm{n}+\alpha+\beta+1),-\mathrm{x} ;(\alpha+1),-\mathrm{N} ; 1)$

where, ${ }_{3} \mathrm{~F}_{2}($.$) is the generalized hypergeometric$ function given by:

${ }_{3} \mathrm{~F}_{2}\left(\mathrm{a}_{1}, \mathrm{a}_{2}, \mathrm{a}_{3} ; \mathrm{b}_{1}, \mathrm{~b}_{2} ; \mathrm{z}\right)=\sum_{\mathrm{k}=0}^{\infty} \frac{\left(\mathrm{a}_{1}\right)_{\mathrm{k}}\left(\mathrm{a}_{2}\right)_{\mathrm{k}}\left(\mathrm{a}_{3}\right)_{\mathrm{k}} \mathrm{z}^{\mathrm{k}}}{\left(\mathrm{b}_{1}\right)_{\mathrm{k}}\left(\mathrm{b}_{2}\right)_{\mathrm{k}} \mathrm{k} !}$

and $(a)_{k}$ is the Pochhammer symbol given by:

$$
(a)_{k}=a(a+1)(a+2) \ldots \ldots .(a+k-1)
$$

$\mathrm{w}(\mathrm{x}, \alpha, \beta, \mathrm{N})$ is the weight function given by:

$$
\mathrm{w}(\mathrm{x} ; \alpha, \beta, \mathrm{N})=\left(\begin{array}{l}
\alpha+\mathrm{x} \\
\mathrm{x}
\end{array}\right)\left(\begin{array}{l}
\beta+\mathrm{N}-\mathrm{x} \\
\mathrm{N}-\mathrm{x}
\end{array}\right)
$$

and the squared norm $\rho(\mathrm{n} ; \alpha, \beta, \mathrm{N})$ is given by:

$$
\rho(n ; \alpha, \beta, N)=\frac{(-1)^{n}(n+\alpha+\beta+1)_{N+1}(\beta+1)_{n} n !}{(2 n+\alpha+\beta+1)(\alpha+1)_{n}(-N)_{n} N !}
$$

Recursive formula for weight function: The weight function as given in (5) can be written as:

$$
\mathrm{w}(\mathrm{x} ; \alpha, \beta, N)=\frac{(\alpha+\mathrm{x}) !(\beta+\mathrm{N}-\mathrm{x}) !}{\mathrm{x} ! \alpha ! \beta !(N-\mathrm{x}) !}
$$

Substituting $\mathrm{x}=\mathrm{x}+1$ in the above equation:

$$
\mathrm{w}(\mathrm{x}+1 ; \alpha, \beta, \mathrm{N})=\frac{(\alpha+\mathrm{x}+1) !(\beta+\mathrm{N}-\mathrm{x}-1) !}{(\mathrm{x}+1) ! \alpha ! \beta !(\mathrm{N}-\mathrm{x}-1) !}
$$

By dividing (8) with (7), we obtain:

$$
\mathrm{w}(\mathrm{x}+1 ; \alpha, \beta, \mathrm{N})=\frac{(\alpha+\mathrm{x}+1)(\mathrm{N}-\mathrm{x})}{(\beta+\mathrm{N}-\mathrm{x})(\mathrm{x}+1)} \mathrm{w}(\mathrm{x} ; \alpha, \beta, \mathrm{N})
$$

The starting value for the above recursion can be obtaining by substituting $\mathrm{x}=0$ in (7) as: $\mathrm{w}(0 ; \alpha, \beta, \mathrm{N})=\frac{(\beta+\mathrm{N}) !}{\beta ! \mathrm{N} !}$

For $\alpha=\beta=0, \mathrm{w}(0 ; \alpha, \beta, \mathrm{N})$ is given by $\mathrm{w}(0 ; \alpha, \beta$, $\mathrm{N})=1$.

Recursive formula for squared norm: By substituting $\mathrm{n}=\mathrm{n}+1$ in (6), we obtain:

$$
\rho(n+1 ; \alpha, \beta, N)=\frac{(-1)^{\mathrm{n}+1}(\mathrm{n}+\alpha+\beta+2)_{\mathrm{N}+1}}{(\beta+1)_{\mathrm{n}+1}(\mathrm{n}+1) !}
$$

Dividing (11) with (6), we obtain the recursive formula for squared norm as:

$$
\begin{aligned}
& \rho(\mathrm{n}+1 ; \alpha, \beta, \mathrm{N})= \\
& (-1)(\mathrm{n}+1)(2 \mathrm{n}+\alpha+\beta+1) \backslash \\
& \frac{(\beta+\mathrm{n}+1)(\mathrm{n}+\alpha+\beta+\mathrm{N}+2)}{(\mathrm{n}-\mathrm{N})(\alpha+\mathrm{n}+1)(\alpha+\beta+\mathrm{n}+1)} \rho(\mathrm{n} ; \alpha, \beta, \mathrm{N}) \\
& \quad(2 \mathrm{n}+\alpha+\beta+3)
\end{aligned}
$$

The starting value for the above recursive relation can be obtaining by substituting $n=0$ in (6) as:

$$
\rho(0 ; \alpha, \beta, N)=\frac{(\alpha+\beta+1)_{N+1}}{(\alpha+\beta+1) N !}
$$

For $\alpha=\beta=0, \rho(0 ; \alpha, \beta, N)$ is given by $\rho(0 ; \alpha, \beta$, $\mathrm{N})=\mathrm{N}+1$.

Recursive formula for weighted Hahn polynomials: The recursive formula for Hahn polynomials with respect to ' $\mathrm{x}$ ' is given by:

$$
\begin{aligned}
& (\mathrm{x}+1-\mathrm{N})(\mathrm{x}+\alpha+2) \mathrm{h}_{\mathrm{n}}(\mathrm{x}+2 ; \alpha, \beta, N) \\
& =\left(4 \mathrm{x}-\beta-\beta \mathrm{x}+2 \mathrm{x}^{2}+2+\mathrm{n}^{2}-3 \mathrm{~N}-\right. \\
& \quad 2 \mathrm{Nx}-\alpha \mathrm{N}+\mathrm{n} \beta+\mathrm{n}+\mathrm{n} \alpha+\alpha+\alpha \mathrm{x}) \mathrm{h}_{\mathrm{n}}(\mathrm{x}+1 ; \alpha, \beta, N) \\
& -(\mathrm{x}+1)(\mathrm{x}-\beta-N) \mathrm{h}_{\mathrm{n}}(\mathrm{x} ; \alpha, \beta, N)
\end{aligned}
$$

for $\mathrm{x}=0,1, \ldots, \mathrm{N}-2$ with $\mathrm{h}_{\mathrm{n}}(0 ; \alpha, \beta, \mathrm{N})=1 \quad$ and $\mathrm{h}_{\mathrm{n}}(1 ; \alpha, \beta, \mathrm{N})=1-\frac{\mathrm{n}(\mathrm{n}+\alpha+\beta+1)}{\mathrm{N}(\alpha+1)}$ as initial values.

In order to derive the recursive formula for weighted Hahn polynomials, we multiply the above equation by: 


$$
\begin{aligned}
& \sqrt{\frac{\mathrm{w}(\mathrm{x}+2 ; \alpha, \beta, \mathrm{N})}{\rho(\mathrm{n} ; \alpha, \beta, \mathrm{N})}}(\mathrm{x}+1-\mathrm{N})(\mathrm{x}+\alpha+2) \mathrm{h}_{\mathrm{n}} \\
& (\mathrm{x}+2 ; \alpha, \beta, \mathrm{N}) \sqrt{\frac{\mathrm{w}(\mathrm{x}+2 ; \alpha, \beta, N)}{\rho(\mathrm{n} ; \alpha, \beta, N)}}=\left(4 \mathrm{x}-\beta-\beta \mathrm{x}+2 \mathrm{x}^{2}\right. \\
& \left.+2+n^{2}-3 \mathrm{~N}-2 \mathrm{Nx}-\alpha \mathrm{N}+\mathrm{n} \beta+\mathrm{n}+\mathrm{n} \alpha+\alpha+\alpha \mathrm{x}\right) \\
& h_{n}(x+1 ; \alpha, \beta, N) \sqrt{\frac{w(x+2 ; \alpha, \beta, N)}{\rho(n ; \alpha, \beta, N)}}-(x+1)(x-\beta-N) \\
& h_{n}(x ; \alpha, \beta, N) \sqrt{\frac{w(x+2 ; \alpha, \beta, N)}{\rho(n ; \alpha, \beta, N)}}=\left(4 x-\beta-\beta x+2 x^{2}\right. \\
& \left.+2+n^{2}-3 \mathrm{~N}-2 \mathrm{Nx}-\alpha \mathrm{N}+\mathrm{n} \beta+\mathrm{n}+\mathrm{n} \alpha+\alpha+\alpha \mathrm{x}\right) \mathrm{h}_{\mathrm{n}} \\
& (\mathrm{x}+1 ; \alpha, \beta, \mathrm{N}) \sqrt{\frac{\mathrm{w}(\mathrm{x}+2 ; \alpha, \beta, \mathrm{N}) \mathrm{w}(\mathrm{x}+1, \alpha, \beta, \mathrm{N})}{\rho(\mathrm{n} ; \alpha, \beta, \mathrm{N}) \mathrm{w}(\mathrm{x}+1, \alpha, \beta, \mathrm{N})}}
\end{aligned}
$$

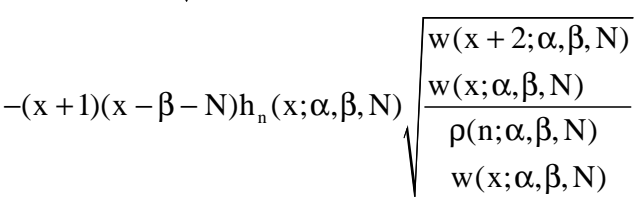

$$
\begin{aligned}
& \Rightarrow(\mathrm{x}+1-\mathrm{N})(\mathrm{x}+\alpha+2) \overline{\mathrm{h}}_{\mathrm{n}}(\mathrm{x}+2 ; \alpha, \beta, \mathrm{N})= \\
& \left(4 x-\beta-\beta x+2 x^{2}+2+n^{2}-3 N-2 N x-\alpha N+n \beta+n\right. \\
& +n \alpha+\alpha+\alpha x) \bar{h}_{n}(x+1 ; \alpha, \beta, N) \sqrt{\frac{\mathrm{w}(\mathrm{x}+2 ; \alpha, \beta, \mathrm{N})}{\mathrm{w}(\mathrm{x}+1, \alpha, \beta, \mathrm{N})}} \\
& -(\mathrm{x}+1)(\mathrm{x}-\beta-\mathrm{N}) \overline{\mathrm{h}}_{\mathrm{n}}(\mathrm{x} ; \alpha, \beta, \mathrm{N}) \sqrt{\frac{\mathrm{w}(\mathrm{x}+2 ; \alpha, \beta, N)}{\mathrm{w}(\mathrm{x} ; \alpha, \beta, N)}}
\end{aligned}
$$

By using the recursive formula for weight function as given in (9):

$$
\begin{aligned}
& \frac{\mathrm{w}(\mathrm{x}+2 ; \alpha, \beta, \mathrm{N})}{\mathrm{w}(\mathrm{x}+1 ; \alpha, \beta, \mathrm{N})}=\frac{(\alpha+\mathrm{x}+2)(\mathrm{N}-\mathrm{x}-1)}{(\beta+\mathrm{N}-\mathrm{x}-1)(\mathrm{x}+2)} \text { and } \\
& \frac{\mathrm{w}(\mathrm{x}+2 ; \alpha, \beta, \mathrm{N})}{\mathrm{w}(\mathrm{x} ; \alpha, \beta, \mathrm{N})}=\frac{(\alpha+\mathrm{x}+2)(\mathrm{N}-\mathrm{x}-1)(\alpha+\mathrm{x}+1)(\mathrm{N}-\mathrm{x})}{(\beta+\mathrm{N}-\mathrm{x}-1)(\mathrm{x}+2)(\beta+\mathrm{N}-\mathrm{x})(\mathrm{x}+1)}
\end{aligned}
$$

Substituting the above values in (15), we obtain the recursive formula for weighted Hahn polynomials as:

$$
\begin{aligned}
& (\mathrm{x}+1-\mathrm{N})(\mathrm{x}+\alpha+2) \overline{\mathrm{h}}_{\mathrm{n}}(\mathrm{x}+2 ; \alpha, \beta, \mathrm{N})= \\
& \left(4 \mathrm{x}-\beta-\beta \mathrm{x}+2 \mathrm{x}^{2}+2+\mathrm{n}^{2}-3 \mathrm{~N}-2 \mathrm{Nx}-\right. \\
& \alpha \mathrm{N}+\mathrm{n} \beta+\mathrm{n}+\mathrm{n} \alpha+\alpha+\alpha \mathrm{x}) \\
& \sqrt{\frac{(\alpha+\mathrm{x}+2)(\mathrm{N}-\mathrm{x}-1)}{(\beta+\mathrm{N}-\mathrm{x}-1)(\mathrm{x}+2)}} \overline{\mathrm{h}}_{\mathrm{n}}(\mathrm{x}+1 ; \alpha, \beta, \mathrm{N})-(\mathrm{x}+1) \\
& (\mathrm{x}-\beta-\mathrm{N}) \sqrt{\frac{(\alpha+\mathrm{x}+2)(\mathrm{N}-\mathrm{x}-1)}{(\beta+\mathrm{x}+1)(\mathrm{N}-\mathrm{x})}}-\overline{\mathrm{h}}_{\mathrm{n}}(\mathrm{x} ; \alpha, \beta, \mathrm{N}) \\
& \sqrt{(\beta+\mathrm{N}-\mathrm{x})(\mathrm{x}+1)(\mathrm{x}+2)}
\end{aligned}
$$

The initial values for the above recursive formula can be obtained using (1) as:

$$
\begin{aligned}
\overline{\mathrm{h}}_{\mathrm{n}}(0 ; \alpha, \beta, \mathrm{N}) & =\mathrm{h}_{\mathrm{n}}(0 ; \alpha, \beta, \mathrm{N}) \sqrt{\frac{\mathrm{w}(0 ; \alpha, \beta, \mathrm{N})}{\rho(\mathrm{n} ; \alpha, \beta, \mathrm{N})}} \\
& =\sqrt{\frac{\mathrm{w}(0 ; \alpha, \beta, \mathrm{N})}{\rho(\mathrm{n} ; \alpha, \beta, \mathrm{N})}}
\end{aligned}
$$

and

$$
\begin{aligned}
\overline{\mathrm{h}}_{\mathrm{n}}(1 ; \alpha, \beta, \mathrm{N}) & =\mathrm{h}_{\mathrm{n}}(1 ; \alpha, \beta, \mathrm{N}) \sqrt{\frac{\mathrm{w}(1 ; \alpha, \beta, \mathrm{N})}{\rho(\mathrm{n} ; \alpha, \beta, \mathrm{N})}} \\
& =\mathrm{h}_{\mathrm{n}}(1 ; \alpha, \beta, \mathrm{N}) \sqrt{\frac{\mathrm{w}(1 ; \alpha, \beta, \mathrm{N}) \mathrm{w}(0 ; \alpha, \beta, \mathrm{N})}{\mathrm{w}(0 ; \alpha, \beta, N) \rho(\mathrm{n} ; \alpha, \beta, N)}}
\end{aligned}
$$

Using (9), we obtain:

$$
\frac{w(1 ; \alpha, \beta, N)}{w(0 ; \alpha, \beta, N)}=\frac{(\alpha+1) N}{\beta+N}
$$

and we have:

$$
h_{n}(1 ; \alpha, \beta, N)=1-\frac{n(n+\alpha+\beta+1)}{N(\alpha+1)}
$$

Substituting the above values in (18), we get:

$$
\begin{aligned}
\overline{\mathrm{h}}_{\mathrm{n}}(1 ; \alpha, \beta, \mathrm{N})= & \left(1-\frac{\mathrm{n}(\mathrm{n}+\alpha+\beta+1)}{\mathrm{N}(\alpha+1)}\right) \\
& \sqrt{\frac{(\alpha+1) \mathrm{N}}{\beta+\mathrm{N}}} \overline{\mathrm{h}}_{\mathrm{n}}(0 ; \alpha, \beta, \mathrm{N})
\end{aligned}
$$

\section{RESULTS}

In order to test the validity of the derived recursive formulas, we calculate Hahn moments $\left(\alpha_{1}=\alpha_{2}=\beta_{1}=\right.$ $\left.\beta_{2}=0\right)$ up to order $(120,120)$ for a Lena image of size $256 \times 256$ as well as Circbw image of size $270 \times 270$. To compute Hahn moments, we use the derived recursive formulas for weight function, squared norm and Weighted Hahn polynomials. Further, we also use the symmetry property of weighted Hahn polynomials and matrix form representation of moments to reduce the computational complexity. The mathematical expression corresponding to symmetry property is given by $\overline{\mathrm{h}}_{\mathrm{n}}(\mathrm{x} ; \alpha, \beta, \mathrm{N}-1)=(-1)^{\mathrm{n}} \overline{\mathrm{h}}_{\mathrm{n}}(\mathrm{N}-1-\mathrm{x} ; \alpha, \beta, \mathrm{N}-1)$. To compute the weighted Hahn polynomials for an image of size $\mathrm{N} \times \mathrm{N}$, one need to compute weighted Hahn polynomials for one quadrant $0 \leq \mathrm{x}, \mathrm{y} \leq(\mathrm{N} / 2-1)$ and 
apply the symmetry property to get the weighted Hahn polynomials for other quadrants. The matrix form representation for Hahn moments is given by:

$$
\mathrm{H}=\mathrm{AFB}^{\mathrm{T}}
$$

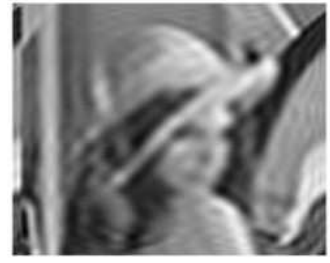

(a)

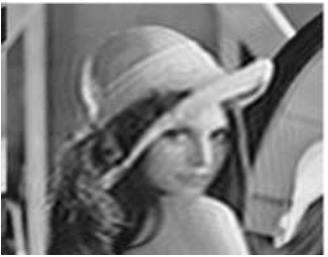

(b)

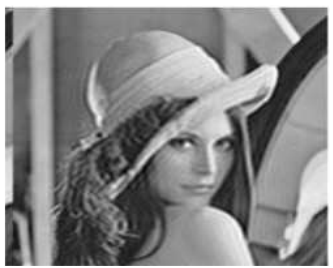

(c)

Fig. 1: Reconstructed Lena images using up to various orders of Hahn moments $\left(\alpha_{1}=\alpha_{2}=\beta_{1}=\beta_{2}=0\right)$ (a) order $(40,40)$ (b) order $(80,80)$ (c) order $(120,120)$

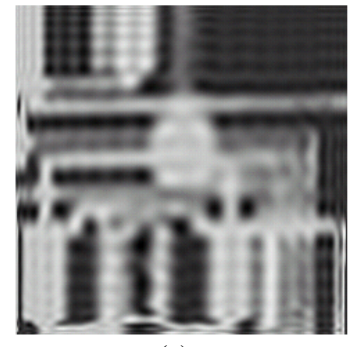

(a)

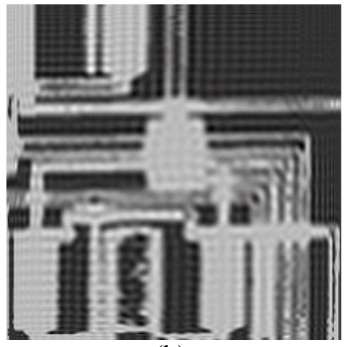

(b)

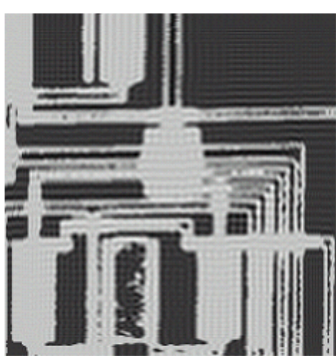

(c)

Fig. 2: Reconstructed Circbw images using up to various orders of Hahn moments $\left(\alpha_{1}=\alpha_{2}=\beta_{1}=\right.$ $\beta_{2}=0$ ) (a) order $(40,40)$ (b) order $(80,80)$ (c) order $(120,120)$ and image reconstruction using Hahn moments is given by:

$$
\mathrm{F}=\mathrm{A}^{\mathrm{T}} \mathrm{HB}
$$

In these expressions, the matrices $\mathrm{H}, \mathrm{A}, \mathrm{B}$ and $\mathrm{F}$ are given by:

$$
\begin{aligned}
& H=\left[\mathrm{H}_{\mathrm{ij}}\right]_{\mathrm{i}, \mathrm{j}=0}^{\mathrm{i}=\mathrm{N}_{\max }, \mathrm{j}=\mathrm{M}_{\max }} \\
& \mathrm{F}=[\mathrm{f}(\mathrm{i}, \mathrm{j})]_{\mathrm{i}, \mathrm{j}=0}^{\mathrm{i}=\mathrm{N}-1, \mathrm{j}=\mathrm{M}-1} \\
& \mathrm{~A}=\left[\overline{\mathrm{h}}_{\mathrm{i}}\left(\mathrm{j} ; \alpha_{1}, \beta_{1}, \mathrm{~N}-1\right)\right]_{\mathrm{i}, \mathrm{j}=0}^{\mathrm{i}=\mathrm{N}_{\max }, \mathrm{j}=\mathrm{N}-1} \\
& \mathrm{~B}=\left[\overline{\mathrm{h}}_{\mathrm{i}}\left(\mathrm{j} ; \alpha_{2}, \beta_{2}, \mathrm{M}-1\right)\right]_{\mathrm{i}, \mathrm{j}=0}^{\mathrm{i}=\mathrm{M}_{\max }, \mathrm{j}=\mathrm{M}-1}
\end{aligned}
$$

$\mathrm{T}$ denotes the transpose of matrix.

The images are reconstructed using Hahn moments up to order $(40,40),(80,80)$ and $(120,120)$ and the reconstructed images are shown in Fig. 1 and 2. In order to assess the quality of reconstructed image, we also compute Universal Image Quality Index (UIQI) (Wang and Bovik, 2002) and the results are entered in Table 1 and 2.

\section{DISCUSSION}

It is observed from the UIQI results that this value approaches to unity as the order of moments used for image reconstruction increases. The image reconstruction is repeated using Hahn moments with parameters $\left(\alpha_{1}=\alpha_{2}=\beta_{1}=\beta_{2}=1\right)$ and the results are shown in Fig. 3 and 4, Table 1 and 2. It is observed that the reconstructed results depend not only on the number of moments selected but also on the parameter values. For the given order of moments, as the value of parameters $\alpha_{1}, \alpha_{2}, \beta_{1}, \beta_{2}$ deviates on either side from unity, the quality of reconstructed image decreases. This is due to the variations in the amplitude of the polynomial values.

Table 2: Computed UIQI for Circbw image

\begin{tabular}{lll}
\hline Order & $\begin{array}{l}\text { UIQI with Hahn moments } \\
\text { for }\left(\alpha_{1}=\alpha_{2}=\beta_{1}=\beta_{2}=0\right)\end{array}$ & $\begin{array}{l}\text { UIQI with Hahn moments } \\
\text { for }\left(\alpha_{1}=\alpha_{2}=\beta_{1}=\beta_{2}=1\right)\end{array}$ \\
\hline$(20,20)$ & 0.06 & 0.06 \\
$(40,40)$ & 0.15 & 0.15 \\
$(60,60)$ & 0.22 & 0.22 \\
$(80,80)$ & 0.29 & 0.30 \\
$(100,100)$ & 0.36 & 0.37 \\
$(120,120)$ & 0.40 & 0.41 \\
\hline
\end{tabular}




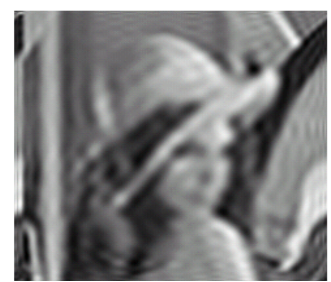

(a)

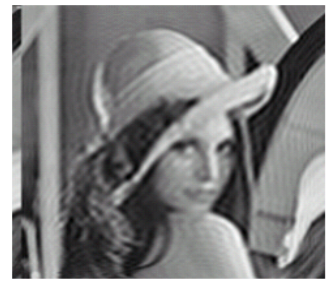

(b)

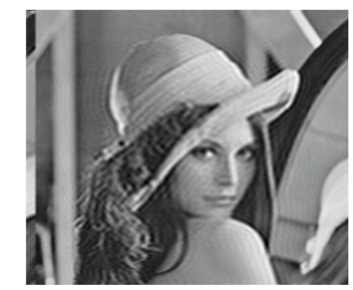

(c)

Fig. 3: Reconstructed Lena images using up to various orders of Hahn moments $\left(\alpha_{1}=\alpha_{2}=\beta_{1}=\beta_{2}=1\right)$ (a) order $(40,40)$ (b) order $(80,80)$ (c) order $(120,120)$

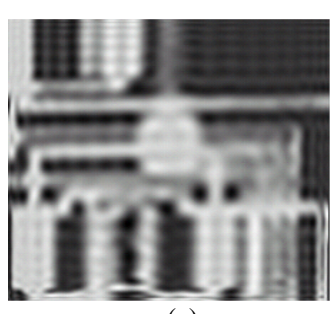

(a)

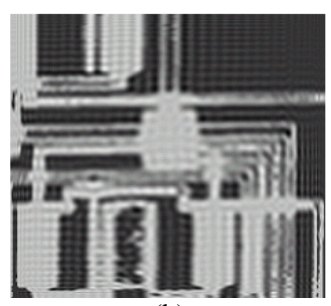

(b)

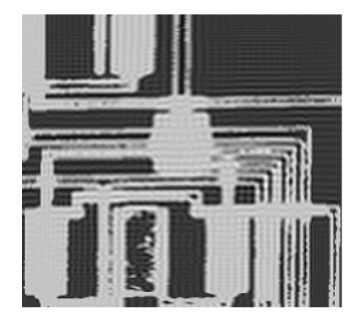

(c)

Fig. 4: Reconstructed Circbw images using up to various orders of Hahn moments $\left(\alpha_{1}=\alpha_{2}=\beta_{1}=\right.$ $\beta_{2}=1$ ) (a) order $(40,40)$ (b) order $(80,80)$ (c) order $(120,120)$

\section{CONCLUSION}

This study presented a procedure for calculation of Hahn moments for any size image using the recursive equations. These equations avoid the need for calculation of factorial of large values and also avoids the numerical instability, which occur in the polynomial calculations.

\section{REFERENCES}

Hu, M.K., 1962. Visual pattern recognition by moment invariants. IRE Trans. Inform. Theory, 8: $179-187$. DOI: $10.1234 / 12345678$

Mukundan, R., S.H. Ong and P.A. Lee, 2001. Image analysis by Tchebichef moments. IEEE Trans. Image Proc., 10: 1357-1363. DOI: $10.1109 / 83.941859$

Teague, M.R., 1980. Image analysis via the general theory of moments. J. Optic. Soc. Am. 70: 920-930. DOI: $10.1364 /$ JOSA.70.000920

Wang, Z. and A.C. Bovik, 2002. A universal image quality index. IEEE Sig. Proc. Lett., 9: 81-84. DOI: 10.1175/JHM563.1

Yap, P.T., R. Parameshan and S.H. Ong, 2003. Image analysis by Krawtchouk moments. IEEE Trans. Image Proc., 12: 1367-1377. DOI: $10.1109 / 2003.43$

Yap, P.T., R. Parameshan and S.H. Ong, 2007. Image analysis using Hahn moments. IEEE Trans. Patt. Anal. Mach. Intel., 29: 2057-2062. DOI: 10.1109/TPAMI.2007.70709 